\title{
Lessons on black holes from the elliptic genus
}

\author{
Amit Giveon, ${ }^{a}$ Nissan Itzhaki ${ }^{b}$ and Jan Troost $^{c}$ \\ ${ }^{a}$ Racah Institute of Physics, The Hebrew University, \\ Jerusalem, 91904, Israel \\ ${ }^{b}$ Physics Department, Tel-Aviv University, \\ Ramat-Aviv, 69978, Israel \\ ${ }^{c}$ Laboratoire de Physique Théorique, Unité Mixte du CNRS et de l'École Normale Supérieure \\ associée à l'Université Pierre et Marie Curie 6, École Normale Supérieure, \\ 24 Rue Lhomond Paris 75005, France \\ E-mail: giveon@phys.huji.ac.il, nitzhaki@post.tau.ac.il, \\ troost@lpt.ens.fr
}

Abstract: We further study the elliptic genus of the cigar $\mathrm{SL}(2, \mathbb{R})_{k} / \mathrm{U}(1)$ coset superconformal field theory. We find that, even in the small curvature, infinite level limit, there are holomorphic and non-holomorphic parts that are due to the discrete states and a mismatch in the spectral densities of the continuum, respectively. The mismatch in the continuum is universal, in the sense that it is fully determined by the asymptotic cylindrical topology of the cigar's throat. Since modularity of the elliptic genus requires both the holomorphic and non-holomorphic parts, the holomorphic term is universal as well. The contribution of the discrete states is thus present even for perturbative strings propagating in the background of large Schwarzschild black holes. We argue that the discrete states live at a stringy distance from the tip of the cigar both from the conformal field theory wave-function analysis and from a holonomy space perspective. Thus, the way string theory takes care of its self-consistency seems to have important consequences for the physics near horizons, even for parametrically large black holes.

Keywords: Conformal Field Models in String Theory, Black Holes in String Theory

ARXIV EPRINT: 1401.3104 


\section{Contents}

1 Introduction $\quad 2$

2 The physics of non-compact elliptic genera 3

2.1 The Witten index and the continuum 3

2.2 The elliptic genus 4

2.3 The cigar conformal field theory 5

$\begin{array}{lll}2.4 & \text { The cigar elliptic genus } & 6\end{array}$

3 The large level limit of the elliptic genus $\quad 6$

$\begin{array}{ll}3.1 & \text { The limit in the Hamiltonian form }\end{array}$

$\begin{array}{lll}3.2 & \text { The identification of states } & 8\end{array}$

$\begin{array}{lll}3.3 & \text { Target-space location } & 9\end{array}$

$\begin{array}{llr}4 & \text { Universality } & 10\end{array}$

5 The saddle point approach $\quad 13$

$\begin{array}{ll}5.1 \text { The holonomy plane } & 13\end{array}$

$\begin{array}{ll}5.2 \text { The saddle point analysis } & 14\end{array}$

$\begin{array}{ll}5.3 \text { The partition function } & 15\end{array}$

$\begin{array}{lll}6 & \text { A UV/IR relation } & 17\end{array}$

$\begin{array}{llr}7 & \text { The trumpet and universality } & 19\end{array}$

$\begin{array}{lll}8 & \text { Summary and discussion } & 20\end{array}$

A Useful formulas $\quad 21$

$\begin{array}{lll}\text { A.1 Conventions and properties of theta functions } & 21\end{array}$

A.1.1 Product and sum formulas 21

$\begin{array}{lll}\text { A.1.2 Properties } & 22\end{array}$

$\begin{array}{lll}\text { A.1.3 Shifts } & 22\end{array}$

$\begin{array}{ll}\text { A.1.4 Derivatives } & 22\end{array}$

A.2 Further properties applied 23

A.2.1 Infinite series, theta functions and a partition sum 23

A.2.2 The expansion of the large level genus near $\alpha=0 \quad 24$ 


\section{Introduction}

Recently [1-3], progress was made in the understanding of the elliptic genus associated with the cigar geometry obtained in the $\mathrm{SL}(2, \mathbb{R})_{k} / \mathrm{U}(1)$ coset superconformal field theory. In this paper, we take advantage of these developments and address a question that was raised in the last year $[4,5]$ : is the tip of the cigar geometry special in the small curvature limit?

From a general relativity perspective, it is hard to see how this can come about, since the curvature and dilaton slope at the tip vanish in the $k \rightarrow \infty$ limit. Nevertheless, motivated by [6], it was argued $[4,5]$ that even in the large $k$ limit there are stringy degrees of freedom with wave-functions that are supported at the tip of the cigar and that fall off exponentially fast at distances larger than the string scale. (See also [7], where the relevance of these degrees of freedom to string theory thermodynamics near the Hagedorn transition was further investigated.) These stringy degrees of freedom are in the discrete representations of the $\mathrm{SL}(2, \mathbb{R})_{k} / \mathrm{U}(1)$ model.

The elliptic genus is an extremely useful tool to carefully address the existence of these states. The reason is that, unlike the partition function, it is free of divergences associated with the infinite volume of the cigar at any finite level $k$ and it gets contributions from the discrete states. Thus, the large $k$ limit of the elliptic genus reveals unambiguously the fate of the discrete states.

In this paper, we take the large level limit of the elliptic genus in two complementary ways. In section 3 , we use the Hamiltonian form and find that the holomorphic piece due to the discrete states is finite even at $k \rightarrow \infty$. The non-holomorphic piece, due to a mismatch in spectral densities between the bosonic and fermionic continuum, is also finite. Aspects of this mismatch are reviewed in section 2. Modularity of the elliptic genus requires the existence of both terms. We conclude that the discrete states survive the large $k$ limit.

Following $[8,9]$, we analyze the target-space wave-functions associated with the discrete states using the $\mathrm{SL}(2, \mathbb{R})_{k} / \mathrm{U}(1)$ Laplacian eigenvalues and find that, indeed, in the large $k$ limit they are located at the tip of the cigar. In section 4 , we argue that the answer obtained for the elliptic genus in the large $k$ limit is universal. That is, provided the nonholomorphic part of the elliptic genus is non-trivial, we expect to find similar states also for cigar geometries associated with other black holes, perhaps including Schwarzschild in four dimensions, in the limit where their mass is parametrically large. It would be good to confirm this intuition through a direct calculation on the Euclidean Schwarschild geometry.

In section 5 , we use the Lagrangian approach to study the large $k$ limit of the elliptic genus. We show that the saddle point approximation is particularly efficient. It also suggests a novel UV/IR relation between the cigar target space and the holonomy space, discussed in section 6 . This UV/IR relation can be used to find the location of states in target space knowing the size of the region in holonomy space that supports them. Using this approach, we confirm that the holomorphic term in the elliptic genus comes from states that live at the tip of the cigar. In section 7 , we refine our discussion of universality via inspection of the elliptic genus of the trumpet conformal field theory. Finally, we conclude in section 8 , and present useful formulas in the appendix. 


\section{The physics of non-compact elliptic genera}

In this section, we present generic features of non-compact elliptic genera. We focus on the physical meaning of the holomorphic anomaly in the non-compact elliptic genus. We start by illustrating that the origin of the anomaly can be traced to the calculation of a Witten index [10] in supersymmetric quantum mechanics with a spectrum that contains both discrete states and a continuum (see e.g. [11]). Then we show that those properties are inherited by a two-dimensional generalization of the Witten index, the elliptic genus. ${ }^{1}$ We review how these features apply to the $\mathrm{SL}(2, \mathbb{R})_{k} / \mathrm{U}(1)$ model. We thus set the stage for a detailed study of the elliptic genus of the $\mathrm{SL}(2, \mathbb{R})_{k} / \mathrm{U}(1)$ coset superconformal field theory at large level in section 3.

\subsection{The Witten index and the continuum}

Consider a supersymmetric quantum mechanics with at least one supersymmetry and a fermion number operator $F$ that takes value 0 on bosons and 1 on fermions. We study the weighted trace $Z$,

$$
Z=\operatorname{Tr}(-1)^{F} e^{-\beta H},
$$

where $H$ is the Hamiltonian, and $\beta$ the inverse temperature of the system. When the spectrum is discrete, the weighted trace is equal to an index, the Witten index [10], that counts the number of bosonic minus the number of fermionic ground states.

When the spectrum of the supersymmetric quantum mechanics contains a continuum, the weighted trace will obtain a contribution from the integral over the continuum, with a measure given by the difference in spectral densities between bosons and fermions (see e.g. [11]),

$$
\operatorname{Tr}(-1)^{F} e^{-\beta H}=N_{\text {bos }}^{\text {ground }}-N_{\text {ferm }}^{\text {ground }}+\int_{\text {cont }} d E\left(\rho_{\text {bos }}(E)-\rho_{\text {ferm }}(E)\right) e^{-\beta E} .
$$

The difference in spectral densities is generically non-trivial, and will contribute a temperature dependent term to the weighted trace. Supersymmetry relates the spectral densities.

Consider for concreteness a non-relativistic supersymmetric quantum mechanics defined on a real line (or half-line) with a scattering wall on one side. Then the difference in spectral densities will be given by a derivative with respect to continuous momentum, $s$, of the ratio of the bosonic and fermionic phase shifts,

$$
\Delta \rho \equiv \rho_{\text {bos }}-\rho_{\text {ferm }}=\frac{1}{2 \pi i} \frac{d}{d s} \log \frac{R_{\text {bos }}}{R_{\text {ferm }}} .
$$

The phase shifts are the logarithms of the corresponding reflection amplitudes $R_{b o s} / \mathrm{ferm}$. Since the asymptotic bosonic and fermionic wave-functions determine the phase shift, and since the asymptotic supercharge takes the bosonic wave-function to the fermionic wavefunction (and vice versa), we see that the difference in spectral densities in these circumstances is determined by the asymptotic supercharge. It can be checked in explicit examples that this expectation is borne out.

\footnotetext{
${ }^{1}$ The properties we present are discussed in references $[1,3,9]$ - it may be convenient to gather them.
} 
Next, we study how this elementary phenomenon in supersymmetric quantum mechanics has far reaching consequences in higher dimensional generalizations of the weighted trace.

\subsection{The elliptic genus}

Elliptic genera of superconformal field theories in two dimensions can be defined as twisted toroidal path integrals in the sector with periodic boundary conditions for the rightmovers [12],

$$
\chi=\operatorname{Tr}_{R}(-)^{\bar{F}} q^{L_{0}-c / 24} \bar{q}^{\bar{L}_{0}-c / 24} x^{Q}
$$

where $q=e^{2 \pi i \tau}$, with $\tau$ being the modular parameter of the torus, and $L_{0}\left(\bar{L}_{0}\right)$ is the left (right)-moving scaling dimension. Here, the right-moving fermion number is denoted $\bar{F}$, and we have introduced a chemical potential $x=e^{2 \pi i \gamma}$ for a $\mathrm{U}(1)$ symmetry $Q$ that commutes with the right-moving supercharge. Since the trace corresponds to a toroidal path integral, it is guaranteed to have good modular covariance properties, as well as good periodicity properties in the twist variable. To define the elliptic genus, it is sufficient to have $(0,1)$ supersymmetry in two dimensions.

As far as the right-moving primaries are concerned, the elliptic genus behaves like a weighted trace in supersymmetric quantum mechanics. As a consequence, when the conformal field theory has a discrete spectrum of right-moving primaries, the elliptic genus projects onto the right-moving ground states. The result is then a holomorphic Jacobi form.

The elliptic genus of theories with a spectrum of conformal dimensions that contains a continuum, obtains contributions from right-moving ground states, as well as from the continuum, due to the difference in the density of bosonic and fermionic right-moving primaries. This difference is determined in terms of the asymptotic right-moving supercharge $[1,9]$. The result is a mock modular contribution from the right-moving ground states, and a modular completion arising from the continuum [1]. These contributions are inextricably linked by modularity and ellipticity $[13,14]$. This mathematical phenomenon has a counterpart in physics, where fixing the asymptotic geometry can lead to a unique filling in in the interior, and thus to a unique set of right-moving ground states.

We wish to comment on an attractive feature of the elliptic genus. Superconformal field theories with continuous spectra exhibit a volume divergence in their partition function. When the global symmetry group is as large as the volume divergence, one can use symmetries to extract the divergent factors, and remain with a calculable and well-defined finite factor. When this is not the case, one can use a twisted partition function, the elliptic genus, to eliminate the volume divergence. The integral over the difference in spectral densities can be finite, even in the presence of infinite volume. Thus, the elliptic genus can serve as a modular covariant infrared regulator.

Next, we review how these generic features are realized in the cigar coset superconformal field theory. 


\subsection{The cigar conformal field theory}

The cigar geometry $[8,15-17]$ and dilaton $\Phi$ associated with the $\mathrm{SL}(2, \mathbb{R})_{k} / \mathrm{U}(1)$ model take the form

$$
d s^{2}=2 k \tanh ^{2}\left(\frac{\rho}{\sqrt{2 k}}\right) d \theta^{2}+d \rho^{2}, \quad \exp (2 \Phi)=\frac{g_{0}^{2}}{\cosh ^{2}\left(\frac{\rho}{\sqrt{2 k}}\right)} .
$$

The angular direction $\theta$ has periodicity $2 \pi$ to ensure smoothness of the background at the tip. We have chosen the convention $\alpha^{\prime}=2$.

Vertex operators in the $\mathrm{SL}(2, \mathbb{R})_{k} / \mathrm{U}(1) N=2$ superconformal field theory are determined by five quantum numbers $\left(n_{f}, \bar{n}_{f}, m, \bar{m}, j\right)$; we have $n_{f}=F-1(1 / 2)$ in the NS (R) sectors, where $F$ is the left-moving worldsheet fermion number, and a similar formula holds for the right-moving fermion numbers; the quantum numbers $m, \bar{m}$ are related to the momentum $p$ and winding $w$ around the angular direction $\theta$ of the cigar via (see e.g. [18] for details)

$$
\left(m+n_{f}, \bar{m}+\bar{n}_{f}\right)=\frac{1}{2}(p+k w,-p+k w), \quad p \in Z+n_{f}-\bar{n}_{f}, w \in Z .
$$

Roughly speaking, the quantum number $j$ is associated with the momentum in the radial direction $\rho$ of the cigar. More precisely, the possible values of $j$ are inherited from either the continuous or the discrete representations of $\operatorname{SL}(2, \mathbb{R})$. In the continuous representations,

$$
j=-1 / 2+i s, \quad s \in \mathbb{R},
$$

while in the discrete representations,

$$
j=|m|-n, \quad n=1,2, \ldots,
$$

and similarly for the right movers. Normalizability of the discrete states implies that they are dominated by their winding contribution, namely,

$$
\operatorname{sign}\left(m+n_{f}\right)=\operatorname{sign}\left(\bar{m}+\bar{n}_{f}\right) .
$$

Moreover, in the quantum theory, we have to impose the unitarity bound $[19,20]$

$$
-\frac{1}{2}<j<\frac{k-1}{2}
$$

The scaling dimension and R-charge are determined by these quantum numbers,

$$
h=\frac{\left(m+n_{f}\right)^{2}-j(j+1)}{k}+\frac{n_{f}^{2}}{2}, \quad R=\frac{2\left(m+n_{f}\right)}{k}+n_{f},
$$

with similar equations for the right movers. Since the $\mathrm{SL}(2, \mathbb{R})_{k} / \mathrm{U}(1)$ conformal field theory contains both discrete and continuous states, the features described in the previous subsections are relevant, and have interesting implications. 


\subsection{The cigar elliptic genus}

In the cigar superconformal field theory, we have additional symmetries compared to the generic case discussed in subsection 2.2. We have a left-moving superconformal algebra with left-moving R-charge $R$ as well as a global U(1) symmetry $P$ corresponding to angular momentum in the direction $\theta$. Since both commute with the right-moving supercharge $\bar{G}_{0}$, they can both be used to twist the elliptic genus. We study the trace with periodic boundary conditions for both left- and right-movers and with the twists

$$
\chi=\operatorname{Tr}_{R}(-)^{F+\bar{F}} q^{L_{0}-c / 24} \bar{q}^{\bar{L}_{0}-c / 24} z^{R} y^{P} .
$$

The operator $F$ denotes left-moving fermion number, while we have fugacities $z=e^{2 \pi i \alpha}$ and $y=e^{2 \pi i \beta}$, where $\alpha$ and $\beta$ are the chemical potentials for the U(1) R-charge and $\theta$ angular momentum $P$, respectively. Far on the cylinder, $\rho \rightarrow \infty$, a good approximation for $P$ is the linear momentum, while near the tip, $\rho \rightarrow 0, P$ is a true angular momentum.

As described in subsection 2.1, the contribution of the continuum is determined in terms of an asymptotic right-moving supercharge, and does not depend on many of the detailed properties of the model. Let's see how this simplification works in practice. For the $\mathrm{SL}(2, \mathbb{R})_{k} / \mathrm{U}(1)$ model, the reflection amplitudes are given by $[19,21,22]$

$$
R(m, \bar{m}, j)=\left(\frac{1}{\pi} \frac{\Gamma\left(1+\frac{1}{k}\right)}{\Gamma\left(1-\frac{1}{k}\right)}\right)^{2 j+1} \frac{\Gamma\left(1-\frac{2 j+1}{k}\right) \Gamma(j+1+m) \Gamma(j+1-\bar{m}) \Gamma(-2 j-1)}{\Gamma\left(1+\frac{2 j+1}{k}\right) \Gamma(m-j) \Gamma(-j-\bar{m}) \Gamma(2 j+1)},
$$

and since $R_{\text {bos }} / R_{\text {ferm }} \equiv R\left(\bar{n}_{f}=-1 / 2\right) / R\left(\bar{n}_{f}=1 / 2\right)$, with $p, w$ and $n_{f}$ held fixed in equation (2.6), one finds for the measure on the real line [3]

$$
\Delta \rho=-\frac{1}{\pi} \frac{1}{2 i s-p+k w} .
$$

Indeed, asymptotically on the cylinder we have the right-moving supercharge $\bar{G}_{0} \sim(2 i s-$ $p+k w)$ in the Ramond sector. ${ }^{2}$ Thus, the spectral asymmetry $\Delta \rho$ is fully determined by the asymptotic right-moving supersymmetry charge $[1,9,11]$. This property will play a key role in the discussion about the universality of our results.

Let us note further that the unique filling in of the internal geometry based on fixed asymptotics, mentioned in subsection 2.2 , is discussed in detail for the cigar case in [23]. ${ }^{3}$

\section{The large level limit of the elliptic genus}

In this section, we analyze the large level limit of the elliptic genus of the cigar conformal field theory. We identify the states that contribute to it, their wave-functions and where they are localized.

\footnotetext{
${ }^{2}$ To be more precise, asymptotically, the right-moving supercurrent can be written as: $\tilde{G}^{+}=\frac{i}{2}\left(\tilde{\psi}_{\rho}+\right.$ $\left.i \tilde{\psi}_{\theta}\right) \partial(\rho-i \theta)+\frac{i}{\sqrt{2 k}} \partial\left(\tilde{\psi}_{\rho}+i \tilde{\psi}_{\theta}\right)$. For large level $k$, the last term drops out. The zero mode sector then gives rise to the right-moving supercharge that we quote, after stripping the fermion zero mode.

${ }^{3}$ Other examples to which similar methods should apply include the asymptotic linear dilaton spaces of $[24]-[28]$.
} 


\subsection{The limit in the Hamiltonian form}

We will calculate the large level $k$ limit of the elliptic genus of the $\mathrm{SL}(2, \mathbb{R})_{k} / \mathrm{U}(1)$ coset model in two ways. The starting point for both is the functional integral expression for the elliptic genus at finite level $k$ that was calculated in [1-3]:

$$
\chi=k \int_{0}^{1} d s_{1} d s_{2} \sum_{m, w \in \mathbb{Z}} \frac{\theta_{11}\left(s_{1} \tau+s_{2}-\alpha(k+1) / k+\beta, \tau\right)}{\theta_{11}\left(s_{1} \tau+s_{2}-\alpha / k+\beta, \tau\right)} e^{2 \pi i \alpha w} e^{-\frac{k \pi}{\tau_{2}}\left|\left(m+s_{2}\right)+\left(w+s_{1}\right) \tau\right|^{2}} .
$$

This result can be understood as arising from a U(1) gauged linear sigma model with one charged $N=(2,2)$ chiral superfield giving rise to the ratio of theta functions, as well as a Stückelberg superfield that cancels the vector multiplet contribution except for their holonomies $s_{1,2}$, and that contributes the last, zero-mode factor depending on windings $m$ and $w$ [26-28]. We set the chemical potential for angular momentum $\beta=0$ in this section. We shall turn it on occasionally to make specific points. Since equation (3.1) arises from path integration over a toroidal worldsheet [1], the functional integral expression is by construction a Jacobi form; that is

$$
\chi(\tau+1, \alpha)=\chi(\tau, \alpha), \quad \text { and } \quad \chi(-1 / \tau, \alpha / \tau)=e^{\pi i \hat{c} \alpha^{2} / \tau} \chi(\tau, \alpha),
$$

with

$$
\hat{c} \equiv c / 3=1+\frac{2}{k} .
$$

A first way to find the large level genus, is to start from the Hamiltonian form of the elliptic genus $\chi$ that is obtained from (3.1) after manipulations that lay bare the contributions as arising from a physical Hilbert space [1-3]. The second way is to take the large $k$ limit directly at the functional integral level (3.1). We follow the latter route in section 5. Each derivation will teach us various physical lessons.

After double Poisson resummation and performing the integrals over the holonomies $s_{1}$ and $s_{2}$, the genus (3.1) can be written in the following way

$$
\chi=\chi^{\text {hol }}+\chi^{\text {rem }}
$$

where $\chi^{\text {hol }}$ is the holomorphic contribution from right-moving ground states and $\chi^{\text {rem }}$ contains non-holomorphic pieces arising from the continuum. They $\operatorname{read}^{4}$

$$
\chi^{\text {hol }}=\frac{i \theta_{11}(z, q)}{\eta^{3}} \sum_{\gamma=0}^{k-1} \sum_{m \in \mathbb{Z}} \frac{q^{k m^{2}-m \gamma} z^{2 m-\frac{\gamma}{k}}}{1-z q^{k m-\gamma}},
$$

and

$$
\chi^{\mathrm{rem}}=-\frac{i \theta_{11}(z, q)}{\pi \eta^{3}} \sum_{p, w} \int_{-\infty-i \epsilon}^{+\infty-i \epsilon} \frac{d s}{2 i s-p+k w} q^{\frac{s^{2}}{k}+\frac{(k w+p)^{2}}{4 k}} z^{\frac{k w+p}{k}} \bar{q}^{\frac{s^{2}}{k}+\frac{(k w-p)^{2}}{4 k}} .
$$

Taking the large $k$ limit of these expressions, one obtains

$$
\chi(k=\infty)=\frac{i \theta_{11}}{\eta^{3}}\left[\frac{1}{2} \frac{1+z}{1-z}+\sum_{n=1}^{\infty}\left(\frac{z q^{n}}{1-z q^{n}}-\left(z \rightarrow z^{-1}\right)\right)+\frac{i \alpha}{2 \tau_{2}}\right] .
$$

\footnotetext{
${ }^{4}$ We consider here the cases with integer level, $k=1,2, \ldots$
} 
Note that this expression is finite at infinite level $k$. Namely, in the large $k$ expansion of the elliptic genus, there are no terms that scale like a positive power of the level $k$. This is an indication that this expression is universal. The non-holomorphic piece, $\frac{i \theta_{11}}{\eta^{3}} \frac{i \alpha}{2 \tau_{2}}$, is due to the continuous representations, as discussed in subsection 2.2. Both the holomorphic and the non-holomorphic contributions are essential for modular covariance. Though this follows from a similar statement at any finite level [1-3], it is also instructive to show this directly on the infinite level expressions.

To demonstrate that the limit elliptic genus (3.7) transforms according to the rules (3.2) with $\hat{c}=1$, we first observe that the holomorphic part of the genus (3.7) can be written in the following form (see useful formulas in the appendix):

$$
\frac{i \theta_{11}}{\eta^{3}}\left[\frac{1}{2} \frac{1+z}{1-z}+\sum_{n=1}^{\infty}\left(\frac{z q^{n}}{1-z q^{n}}-\left(z \rightarrow z^{-1}\right)\right)\right]=-\frac{1}{2 \pi} \frac{\partial_{\alpha} \theta_{11}}{\eta^{3}} .
$$

Consider the modular S-transformation of the holomorphic contribution:

$$
-\frac{1}{2 \pi} \frac{\partial_{\alpha} \theta_{11}(z, q)}{\eta^{3}} \rightarrow-\frac{1}{2 \pi} e^{\pi i \frac{\alpha^{2}}{\tau}} \frac{\partial_{\alpha} \theta_{11}(\alpha, \tau)}{\eta^{3}}-\frac{\alpha}{\tau} e^{\pi i \frac{\alpha^{2}}{\tau}} \frac{i \theta_{11}(\alpha, \tau)}{\eta^{3}} .
$$

The anomalous transformation of the non-holomorphic term in equation (3.7),

$$
\frac{i \theta_{11}}{\eta^{3}} \frac{i \alpha}{2 \tau_{2}} \rightarrow e^{\pi i \frac{\alpha^{2}}{\tau}} \frac{i \theta_{11}}{\eta^{3}} \frac{i \alpha}{2 \tau_{2}} \frac{\bar{\tau}}{\tau}=e^{\pi i \frac{\alpha^{2}}{\tau}} \frac{i \theta_{11}}{\eta^{3}} \frac{i \alpha}{2 \tau_{2}}+e^{\pi i \frac{\alpha^{2}}{\tau}} \frac{i \theta_{11}}{\eta^{3}} \frac{\alpha}{\tau}
$$

exactly cancels the second term in the right hand side of transformation rule (3.9), and renders the genus (3.7) modular covariant. Indeed, the derivative we take can be viewed as a modular covariant derivative acting on a Jacobi form. This is an elementary incarnation of how shadows and mock Jacobi forms are bijectively linked in favorable circumstances $[13,14]$.

\subsection{The identification of states}

We saw that the holomorphic piece of the elliptic genus of the cigar at $k \rightarrow \infty$ takes the form

$$
\chi^{\text {hol }}=\frac{i \theta_{11}(z, q)}{\eta^{3}}\left[\frac{1}{2} \frac{1+z}{1-z}+\sum_{n=1}^{\infty}\left(\frac{z q^{n} y^{n}}{1-z q^{n}}-\left(z \rightarrow z^{-1}, y \rightarrow y^{-1}\right)\right)\right] .
$$

Here we have added in the dependence on the chemical potential for momentum along the angular direction $\theta$. This gives extra information helpful in identifying the states that contribute.

The various terms in equation (3.11) amount to the modules of states that are rightmoving ground states in the Ramond sector, and which survive the $k \rightarrow \infty$ limit. Concretely, in the notation of subsection 2.3 , these are discrete states with quantum numbers $\left(n_{f}, \bar{n}_{f}, m, \bar{m}, j\right)$, satisfying $|\bar{m}|=j+1$. In terms of the asymptotic winding $w$ on the cigar's throat, the only right-moving ground states which do not decouple in the large level limit are winding plus or minus one states. 
States with $w=1, p=1,2, \ldots$ and $\bar{n}_{f}=-1 / 2$ give rise to the $\frac{i \theta_{11}}{\eta^{3}} \sum_{n=1}^{\infty} \frac{z y^{n} q^{n}}{1-z q^{n}}$ term in the elliptic genus, where the integer $p=n=1,2, \ldots$ in the sum is the momentum on the cigar, for the asymptotic winding one states, and the theta-function factor, $i \theta_{11}=\sum_{n_{f} \in \mathbb{Z}+1 / 2}(-1)^{n_{f}+1 / 2} q^{n_{f}^{2} / 2} z^{n_{f}}$, is a sum over the left-moving fermion number, $n_{f}$. The conjugates of these right-moving ground states, those with winding $w=-1$ and momentum $p=-n=-1,-2, \ldots$, as well as opposite right-moving fermion number, $\bar{n}_{f}=1 / 2$, contribute to the term in the elliptic genus obtained by mapping $z \rightarrow z^{-1}$ and $y \rightarrow y^{-1}$.

We can also interpret these contributions as arising from $N=2$ superconformal characters at central charge $c=3$ and with quantum numbers $Q= \pm 1+n_{f}$ and $h= \pm p+c / 24$, along the lines described in [5].

The Hamiltonian interpretation of the term $\frac{i \theta_{11}}{\eta^{3}} \frac{1}{2} \frac{1+z}{1-z}$ in the elliptic genus is more subtle, since as it stands it contains half-integer multiplicities. The subtlety arises from the ambiguity in interpreting a state at the bottom of the continuum as a right-moving ground state. We rewrite this term as

$$
\frac{i \theta_{11}}{\eta^{3}} \frac{1}{2} \frac{1+z}{1-z}=\frac{i \theta_{11}}{\eta^{3}} \frac{z}{1-z}+\frac{1}{2} \frac{i \theta_{11}}{\eta^{3}} .
$$

We can interpret the first contribution as arising from a primary with $w=1$ and zero angular momentum $p=0$. It is a marginal winding tachyon - the $N=2$ Liouville operator, combined via FZZ duality with the dilaton deformation (see e.g. [29] and references therein for details). The second contribution is the result of integration over the continuum of states, and in particular is associated to a choice of regularization of this integral at zero radial momentum $s=0$. To arrive at our Hamiltonian interpretation of the last term, we broke $\alpha \leftrightarrow-\alpha$ charge conjugation symmetry. Another, charge conjugate Hamiltonian interpretation is equally possible.

\subsection{Target-space location}

We focus now on the target-space interpretation of the primary states. In particular, we determine whether these states are located near the string scale tip of the cigar, $\rho \lesssim 1$, or at the cap of the cigar and the regime where it is glued to the asymptotic cylindrical throat, $\rho \lesssim \sqrt{k}$ (see figure 1 ). There are two ways to tackle this important issue. The first is direct, by computing wave-functions corresponding to the states. The second is indirect, by analyzing the localization of the state contributions in the holonomy plane, as we will do in section 6 .

In the previous section, we found the $\mathrm{SL}(2, \mathbb{R})_{k} / \mathrm{U}(1)$ coset quantum numbers associated with the primary states that contribute to the holomorphic term in the elliptic genus. With this information at hand, we can translate the conformal dimension into a Laplacian eigenvalue for the Laplacian on the exact supersymmetric cigar background (2.5) (see e.g. [8] for the application of this technique to the bosonic black hole). We find the target-space wave-function of our states using their conformal dimension ${ }^{5}$

$$
L_{0}|\Psi\rangle=h|\Psi\rangle \quad \text { and } \quad \bar{L}_{0}|\Psi\rangle=\bar{h}|\Psi\rangle
$$

\footnotetext{
${ }^{5}$ Here we work in the NS-NS sector with $n_{f}=\bar{n}_{f}=0$.
} 
with scaling operators

$$
L_{0}=-\frac{1}{k}\left(\triangle-m^{2}\right), \quad \bar{L}_{0}=-\frac{1}{k}\left(\triangle-\bar{m}^{2}\right),
$$

where the $\operatorname{SL}(2, \mathbb{R})$ Laplacian $\triangle$ takes the form ${ }^{6}$

$$
\triangle=\frac{k}{2} \partial_{\rho}^{2}+\sqrt{k / 2} \operatorname{coth}\left(\frac{\rho}{\sqrt{k / 2}}\right) \partial_{\rho}-\frac{1}{\sinh ^{2}\left(\frac{\rho}{\sqrt{k / 2}}\right)}\left(m^{2}+\bar{m}^{2}-2 \cosh \left(\frac{\rho}{\sqrt{k / 2}}\right) m \bar{m}\right) .
$$

We concentrate on the winding numbers $|w|=1$ corresponding to the BPS primaries that survive the infinite level limit. Taking the large $k$ limit at fixed $\rho$ of the condition

$$
\left(L_{0}+\bar{L}_{0}\right)|\Psi\rangle=(h+\bar{h})|\Psi\rangle,
$$

we find that the BPS primary states with $|w|=1$ satisfy the following equation

$$
\left(-\partial_{\rho}^{2}-\frac{1}{\rho} \partial_{\rho}+\frac{\rho^{2}}{4}-1+\frac{p^{2}}{\rho^{2}}\right)\left|\Psi_{p}\right\rangle=|p|\left|\Psi_{p}\right\rangle .
$$

This equation describes a two-dimensional harmonic oscillator with frequency 1 and angular momentum $L=p$ (see [5] for more detail). The difference $L_{0}-\bar{L}_{0}$ is fixed to $h-\bar{h}=$ $p w=|p|$. In terms of the energy, $E$, and angular momentum, $L$, we see that states that contribute to the elliptic genus (3.11) have

$$
E=|L|=n=0,1,2, \ldots
$$

Since the level $k$ dropped out of equation (3.17), it follows that these states are localized at the tip of the cigar.

We conclude that modularity of the elliptic genus implies the existence of these discrete states which in turn makes the origin of $\mathbb{R}^{2}$, as obtained by taking the infinite level limit of the cigar, special. In ordinary flat space $\mathbb{R}^{2}$, all states with definite left and right conformal dimension $h$ and $\bar{h}$ are smeared all over the two-plane. Equation (3.17), governing our wave-functions, implies that at the tip of the cigar there are states with fixed left- and right-moving conformal dimensions that are localized at the string scale.

\section{Universality}

As explained in section 2, the non-holomorphic term in the elliptic genus is due to a mismatch, $\Delta \rho$, between the densities of worldsheet bosonic and fermionic degrees of freedom in the continuum. In the limit $k \rightarrow \infty$, locally the difference in densities approaches zero, $\Delta \rho \sim 1 / k$, but since the area of the cigar's cap blows up, $V_{\text {cap }} \sim k$, there is a finite contribution that scales like $\alpha / \tau_{2}$. Hence, from the target-space point of view the nonholomorphic piece is an infrared effect. Moreover, as emphasized in section 2, the spectral

\footnotetext{
${ }^{6}$ Alternatively, we could directly use the Laplacian on the cigar geometry. Here, we take a coset conformal field theory point of view, i.e. we take the parent conformal field theory as our starting point.
} 
asymmetry $\Delta \rho$ is completely determined by supersymmetry and the asymptotic geometry; that is by the topology of the cigar and not by the details of how its geometry is filled in. Hence, it is natural to expect this large $k$ non-holomorphic piece to be universal.

The topology of the cigar also dictates that the Witten index, $\chi(\alpha=0)$, should be equal to 1. As implied from the discussion around equation (3.12), this too is due to a supergravity mode - the multiplet of the zero mode of the dilaton. Thus, as expected, all aspects of the elliptic genus that are determined solely by the topology are controlled by infrared physics in the target space.

Modularity and the non-holomorphic term determine the holomorphic term. As discussed in the previous sections, this term is due to discrete states that are located at the tip of the cigar. Namely, from the target-space point of view this contribution is a UV effect. Thus, the elliptic genus is another example where a modular transformation mixes between UV and IR degrees of freedom in the target space.

Since the non-holomorphic term is universal and since it determines the holomorphic piece via modularity, we conclude that the holomorphic piece is universal as well. In particular, we expect to find discrete states at the tip associated with generic large mass black holes.

The way the holomorphic term was obtained in the previous section may look nonuniversal; it is a particular sum of characters of the $N=2$ superconformal algebra. This sum is restricted at large level $k$ to asymptotic winding (plus or minus) one states and includes the denominators that are induced by their null state structure.

In the rest of this section, we review an alternative route to modeling the elliptic genus of the cigar theory [9], in terms of a supersymmetric quantum mechanics that arises after Scherk-Schwarz reduction [30] of the $(2,2)$ supersymmetric sigma model on the angular direction. It may shed light on the universal nature of the result. In [9], two counting functions were matched. On the one hand, the elliptic genus after factoring out a generic oscillator contribution $i \theta_{11} / \eta^{3}$ corresponding to all generators of the $N=2$ superconformal algebra, and on the other hand, the number of right-moving ground states as coded in the supersymmetric quantum mechanics corresponding to given winding $w^{\prime}$, and with momentum $p$.

Technically, the right-moving ground states contribution to the elliptic genus was found to be of the form

$$
\chi^{\mathrm{hol}}=\frac{i \theta_{11}}{\eta^{3}}\left(\sum_{p \geq 0,-p+k w^{\prime} \geq 0}-\sum_{p \leq-1,-p+k w^{\prime} \leq-1}\right) z^{\frac{p+k w^{\prime}}{k}} q^{p w^{\prime}} y^{p},
$$

which matches the expression (3.5) for the elliptic genus under the identifications

$$
\begin{aligned}
p & =k m-\gamma, \\
w^{\prime} & =m+r,
\end{aligned}
$$

where $r$ is a quantum number arising from expanding the null vector denominator in our $N=2$ superconformal characters in the regime $|q|<|z|<1$, and $m$ and $\gamma$ are the quantum 
numbers appearing in equation (3.5). In the large level limit, and ignoring ambiguous contributions at zero winding or zero momentum, we then find the expression

$$
\begin{aligned}
\chi^{\text {hol }} & =\frac{i \theta_{11}}{\eta^{3}}\left(\sum_{p \geq 1, w^{\prime}>0}-\sum_{p \leq-1, w^{\prime} \leq-1}\right) z^{w^{\prime}} q^{p w^{\prime}} y^{p} \\
& =\frac{i \theta_{11}}{\eta^{3}}\left(\sum_{p \geq 1} \frac{z q^{p} y^{p}}{1-z q^{p}}-\sum_{p \geq 1} \frac{z^{-1} q^{p} y^{-p}}{1-z^{-1} q^{p}}\right) .
\end{aligned}
$$

We conclude that the states with winding $w=+1$ on the cigar are captured by the states with positive momentum $p \geq 1$ and any positive winding $w^{\prime} \geq 1$ in the supersymmetric quantum mechanics (and a similar statement holds for the charge conjugate states). In the supersymmetric quantum mechanics, we ignore the existence of the fermionic null vector of the $N=2$ superconformal algebra, effectively adding back in a fermionic degree of freedom. In the supersymmetric partition function, this is compensated by adding in a bosonic degree of freedom as well, that gives rise to the infinite sum over winding $w^{\prime}$.

This embedding of the calculation of the elliptic genus directly in flat space may provide an important hint towards finding a universal $c=3, N=2$ conformal field theory that describes string theory at the tip of the cigar.

We also wish to revisit the issue of the size and location in the target space in the framework of the supersymmetric quantum mechanics. In [9], target-space wave-functions for all these states in the NS-R sector were found by solving the right-moving ground state Dirac equation $\bar{G}_{0}|\Psi\rangle=0$ at any finite level. We can take the large level limit on those wave-functions to find that they localize on a disk of string scale size in agreement with the above discussion.

Alternatively, we can work in the NS-NS incarnation of the quantum mechanics problem by solving the Klein-Gordon equation

$$
\left(-\partial_{\rho}^{2}-\frac{1}{\rho} \partial_{\rho}+\frac{w^{\prime 2}}{4} \rho^{2}-1+\frac{p^{2}}{\rho^{2}}\right)\left|\Psi_{w^{\prime}, p}\right\rangle=p w^{\prime}\left|\Psi_{w^{\prime}, p}\right\rangle,
$$

to reach the same conclusion. Here, $L_{0}-\bar{L}_{0}=p w^{\prime}$, and we have used the Laplace operator (3.15) for a BPS primary state with generic winding number $w^{\prime}$.

Universality and Euclidean Schwarzschild. A particularly interesting case in which we would like to apply the concept of universality is that of a large mass $M$ limit of the Euclidean Schwarzschild black hole. When concentrating on the degrees of freedom that do not depend on the two-sphere section (namely, when concentrating on the s-wave, and neglecting string oscillations on the two-sphere), we find again a cigar geometry. We may then expect to find a similar non-holomorphic piece, arising from the gluing of the cap regime to the asymptotic cylindrical throat shape (see figure 1). It would be dictated by the right-moving supercharge at infinity on the Schwarzschild geometry. Once we fixed the nonholomorphic piece, worldsheet modularity implies that the holomorphic piece is universal as well. Namely, the set of right-moving ground states will be fixed, and may contain a spectrum of discrete states localized near the tip, that is near the Lorentzian horizon. 


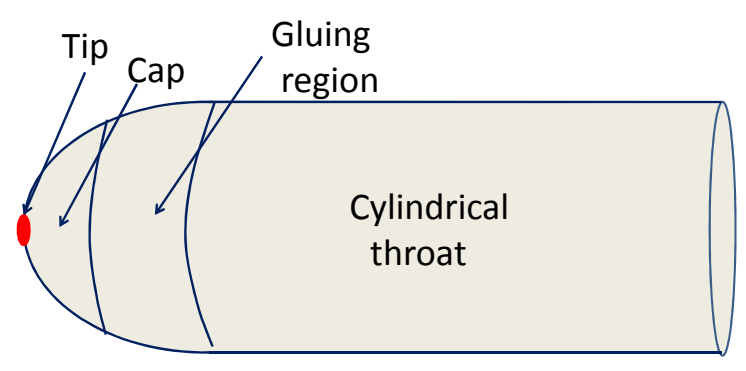

Figure 1. In stringy units, the size of the tip is order 1 , and the size of the cap of the $\operatorname{SL}(2, \mathbb{R})_{k} / \mathrm{U}(1)$ (mass $M$ Schwarzschild) cigar is of order $\sqrt{k}(M)$. A rough definition of the cap is the region at which the curvature (in the string frame) is of order $1 / \sqrt{k}(1 / M)$. Deep in the semi-infinite cylindrical throat the curvature vanishes. The drop in curvature from $1 / \sqrt{k}(1 / M)$ to zero happens exponentially fast in the gluing region. The universality discussed in this section refers to the cap, which in the large $k(M)$ limit looks like $\mathbb{R}^{2}$ with the addition of states that live at the tip and a small mismatch in the continuous spectrum, $\Delta \rho$.

More speculatively still, we may define an analog of the elliptic genus for a $(0,1)$ twodimensional conformal field theory on the Euclidean Schwarzschild background at finite mass $M$. The Ricci flat geometry is reliable at leading order in $1 / M$. For this model, there is a twisted trace of the type reviewed in subsection 2.2 [12]. It would be interesting to confirm our intuition and compute explicitly the contribution to such a twisted trace from the continuum and the degrees of freedom associated to the asymptotic geometry, including the two-sphere, at finite mass. By combining modularity (i.e. universality of the modular completion), as well as a unique filling in of the asymptotics with given topology and satisfying Einstein's equations, one may be able to draw far reaching conclusions on degrees of freedom localized in the deep interior, near the tip. It would be interesting to execute this program in detail.

\section{The saddle point approach}

In the previous section, we analyzed the large level limit of the cigar genus in a Hamiltonian approach. In this section, we will take the limit in a Lagrangian formalism, through saddle point analysis. This approach sheds light, amongst other things, on the fact that the holomorphic piece of the elliptic genus can be written as a derivative in the large level limit.

\subsection{The holonomy plane}

Periodicity properties of theta functions (see the appendix), allow us to extend the range of integration over the holonomies $s_{1}$ and $s_{2}$ in equation (3.1) to obtain

$$
\chi=k \int_{-\infty}^{+\infty} d s_{1} d s_{2} \frac{\theta_{11}\left(s_{1} \tau+s_{2}-\alpha-\alpha / k, \tau\right)}{\theta_{11}\left(s_{1} \tau+s_{2}-\alpha / k, \tau\right)} e^{-\frac{k \pi}{\tau_{2}}\left|s_{2}+s_{1} \tau\right|^{2}} .
$$

In the large $k$ limit, we can do a saddle point approximation to the integral. We must be careful in evaluating the potential large $k$ dependence of the $\theta_{11}$ functions. To that end, 
we redefine the integration variables,

$$
s_{1} \tau+s_{2}=x_{1}+i x_{2},
$$

and pick up the measure factor $\tau_{2}$,

$$
\chi=\frac{k}{\tau_{2}} \int_{-\infty}^{+\infty} d^{2} x \frac{\theta_{11}\left(x_{1}+i x_{2}-\alpha-\alpha / k, \tau\right)}{\theta_{11}\left(x_{1}+i x_{2}-\alpha / k, \tau\right)} e^{-\frac{k \pi}{\tau_{2}}\left(x_{1}^{2}+x_{2}^{2}\right)} .
$$

Rescaling the holonomy plane variables, we get

$$
\chi=\int_{-\infty}^{+\infty} d^{2} x \frac{\theta_{11}\left(\sqrt{\frac{\tau_{2}}{k}}\left(x_{1}+i x_{2}\right)-\alpha-\alpha / k, \tau\right)}{\theta_{11}\left(\sqrt{\frac{\tau_{2}}{k}}\left(x_{1}+i x_{2}\right)-\alpha / k, \tau\right)} e^{-\pi\left(x_{1}^{2}+x_{2}^{2}\right)} .
$$

This expression is a good starting point of the saddle point analysis we perform below.

\subsection{The saddle point analysis}

In the large $k$ limit, we can expand the $\theta$ functions in the denominator and numerator to get

$$
\chi=\int d^{2} x \frac{\theta_{11}(\alpha, \tau)-\left(\sqrt{\frac{\tau_{2}}{k}}\left(x_{1}+i x_{2}\right)-\alpha / k\right) \partial_{\alpha} \theta_{11}(\alpha, \tau)+\ldots}{2 \pi\left(\sqrt{\frac{\tau_{2}}{k}}\left(x_{1}+i x_{2}\right)-\alpha / k+\ldots\right) \eta^{3}} e^{-\pi\left(x_{1}^{2}+x_{2}^{2}\right)} .
$$

The validity of the expansion implies that the range of integration is $x_{1}^{2}+x_{2}^{2}<\frac{k}{\tau_{2}}$. In the large $k$ limit, however, the exponential suppression renders this bound irrelevant and the range of integration is the whole $\left(x_{1}, x_{2}\right)$ plane. The leading term scales like $\sqrt{k}$,

$$
\chi_{\text {lead }}=\sqrt{\frac{k}{\tau_{2}}} \frac{\theta_{11}(\alpha, \tau)}{\eta^{3}} \int \frac{d^{2} x}{x_{1}+i x_{2}} e^{-\pi\left(x_{1}^{2}+x_{2}^{2}\right)} .
$$

This term, however, vanishes because of the integration over the angular direction. Namely, writing $x_{1}+i x_{2}=r e^{i \theta}$, we see that the integral over the angle $\theta$ vanishes.

At the next order we find two terms. The simplest one is from the second term in the numerator,

$$
\chi_{1}=-\frac{\partial_{\alpha} \theta_{11}(\alpha, \tau)}{2 \pi \eta^{3}} \int d^{2} x e^{-\pi\left(x_{1}^{2}+x_{2}^{2}\right)}=-\frac{\partial_{\alpha} \theta_{11}(\alpha, \tau)}{2 \pi \eta^{3}} .
$$

This term is nothing but the holomorphic part of the genus (3.7). Note that the contribution to this term comes from a region of radius of order 1 in the $\left(x_{1}, x_{2}\right)$ plane.

The second contribution comes from the first term in the numerator and a little disk of size $\alpha / \sqrt{k \tau_{2}}$ around the origin. For this contribution, we have

$$
r<\alpha / \sqrt{k \tau_{2}} .
$$

That integral, after expanding the denominator in this region, boils down to

$$
\begin{aligned}
-2 \pi \int_{0}^{\frac{\alpha}{\sqrt{k \tau_{2}}}} d r r \frac{k}{\alpha} e^{-\pi r^{2}} & =\frac{k}{\alpha}\left(-1+e^{-\frac{\pi \alpha^{2}}{k \tau_{2}}}\right) \\
& \rightarrow-\frac{\alpha \pi}{\tau_{2}},
\end{aligned}
$$


such that we get a contribution

$$
\chi_{\mathrm{non}-\mathrm{hol}}=\frac{\theta_{11}}{\eta^{3}}\left(-\frac{\alpha}{2 \tau_{2}}\right),
$$

which is the non-holomorphic term in the genus (3.7). It can be checked that the other region of integration (for radii larger than the critical radius $\alpha / \sqrt{k \tau_{2}}$ ) does not contribute due to the angular integration.

The regions in the holonomy plane that contribute to the two terms in the elliptic genus demand a physical interpretation, perhaps in terms of a model living on the holonomy plane. We will come back to this topic shortly.

We note that the saddle point approach can be used as a technique to tackle many questions in the $\mathrm{SL}(2, \mathbb{R})_{k} / \mathrm{U}(1)$ conformal field theory directly at large level. This includes the calculation of correlation functions, and also the partition function that we turn to next.

\subsection{The partition function}

In this subsection, we use the saddle point approach to analyze the partition function at large level. Our main goal here is to extract more information about the discrete states in the large $k$ limit. (The elliptic genus is sensitive only to those discrete states that are right-moving ground states).

There is another reason the partition function is useful in the study of the large level limit of the $\mathrm{SL}(2, \mathbb{R})_{k} / \mathrm{U}(1)$ model; it is modular invariant. This entails that expanding the partition function in $1 / k$ yields modular invariant expressions at each order.

The functional integral representation of the partition function, including extra fermionic degrees of freedom of a ten-dimensional superstring theory is $[31]^{7}$

$$
\begin{aligned}
Z_{\sigma_{L}, \sigma_{R}}= & k \sum_{m_{i} \in \mathbb{Z}} \int_{0}^{1} d s_{1} d s_{2} \epsilon\left(\sigma_{L} ; m_{1}, m_{2}\right) \epsilon\left(\sigma_{R} ; m_{1}, m_{2}\right) \times \\
& f_{\sigma_{L}}\left(s_{1} \tau+s_{2}, \tau\right) f_{\sigma_{R}}^{*}\left(s_{1} \tau+s_{2}, \tau\right) e^{-\frac{\pi k}{\tau_{2}}\left|\left(s_{1}+m_{1}\right) \tau+\left(s_{2}+m_{2}\right)\right|^{2}} .
\end{aligned}
$$

The left- and right-handed spin structures, $\sigma_{L}, \sigma_{R}$, run over the four sectors $N S, N S, R$ and $\tilde{R}$. The functions $f_{\sigma}$ are given in terms of standard theta functions via

$$
f_{\sigma}(u, \tau)=\frac{\theta_{\sigma}(u, \tau)}{\theta_{1}(u, \tau)}\left(\frac{\theta_{\sigma}(0, \tau)}{\eta(\tau)}\right)^{3},
$$

where $\theta_{\sigma} \equiv \theta_{1,2,3,4}$ for $\sigma=\tilde{R}, R, N S, N S$, respectively, and

$$
\epsilon= \begin{cases}1, & \sigma=N S, \\ (-)^{m_{1}+1}, & \sigma=N S \\ (-)^{m_{2}+1}, & \sigma=R, \\ (-)^{m_{1}+m_{2}}, & \sigma=\tilde{R} .\end{cases}
$$

\footnotetext{
${ }^{7}$ For concreteness, we consider here e.g. the near horizon Euclidean geometry associated with $k$ near extremal NS5-branes in the type II superstring; see e.g. [5, 31] for details.
} 


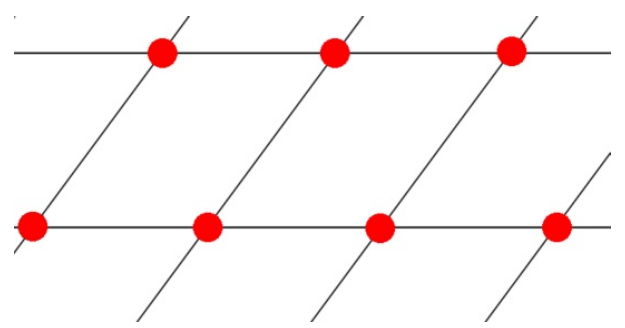

Figure 2. We cut out little disks around lattice points in the holonomy plane to regularize modular invariantly.

Formally, this expression is modular invariant. However, it is divergent at $s_{1,2}=0,1$. The target-space origin of this divergence is the infinite volume of the cylinder [32]. The infinite volume is an obstruction to cleanly dissecting various contributions to the partition function (see e.g. [33] for related puzzles) in contrast to the elliptic genus analysis. In the elliptic genus, the volume divergence is canceled by a (twisted) right-moving fermionic zero mode [1]. Operationally, a $\theta_{1}$ function in the denominator is canceled by an identical $\theta_{1}$ function in the numerator. What remains is a finite integral.

Below, we follow a different way to regularize the partition function, which is modular invariant. Using similar manipulations as in subsection 5.1 (based on equation (A.4)), we put equation (5.11) in the following form

$$
\begin{aligned}
Z_{\sigma_{L}, \sigma_{R}}= & k \int_{-\infty}^{+\infty} d s_{1} d s_{2} \epsilon\left(\sigma_{L}\right) \epsilon\left(\sigma_{R}\right) \times \\
& f_{\sigma_{L}}\left(s_{1} \tau+s_{2}, \tau\right) f_{\sigma_{R}}^{*}\left(s_{1} \tau+s_{2}, \tau\right) e^{-\frac{\pi k}{\tau_{2}}\left|s_{1} \tau+s_{2}\right|^{2}},
\end{aligned}
$$

where $\epsilon=1$ for $\sigma=N S, \tilde{R}$, while $\epsilon=-1$ in the $\tilde{N} S, R$ sectors. This expression diverges when the holonomies take the values

$$
s_{1} \tau+s_{2}=m_{1} \tau+m_{2} .
$$

To deal with these infinities, we cut out from the $x_{1}+i x_{2}=s_{1} \tau+s_{2}$ plane, disks of radius $\varepsilon$ around each of these divergent contributions; see figure 2. This is modular invariant, since under modular transformations each disk transforms into another cutout. This technique contrasts with previous regularization schemes that break modular invariance. ${ }^{8}$

After introducing this regularization, we find that the divergence at the origin gives rise to the contribution

$$
Z_{\text {origin }}=\frac{k}{\tau_{2}} \log (\varepsilon) \frac{\left|\left(\theta_{3}^{4}-\theta_{4}^{4}-\theta_{2}^{4}\right)\right|^{2}}{|\eta|^{12}},
$$

which is modular invariant. It is the expected leading term one gets when considering a target space with a negligible curvature. The divergence is due to states in the continuum

\footnotetext{
${ }^{8}$ Indeed, the region excised in [32] and all follow-ups is a sliver along one side of the torus. This cut is not invariant under modular transformations. It does have the advantage of giving rise to a strict implementation of the coset Gauss law constraint.
} 
with zero winding that propagate along the semi-infinite cylinder. The target-space details of the cut-off are not clear, but very roughly speaking, $\varepsilon$ cuts off the size of the semi-infinite cylinder, a distance of order $\sqrt{k} \log (\varepsilon)$ from the tip. Corrections to the contribution (5.16) that scale like the volume of the cigar are obtained through evaluating the other divergences at the non-zero poles in equation (5.15), that yield terms that scale like, schematically,

$$
Z_{w} \sim k \log (\varepsilon) e^{-\pi \tau_{2} k w^{2}}
$$

These correspond to states in the continuous representations with $w \neq 0$, that propagate along the semi-infinite cylinder.

One question is whether there are corrections to the leading terms, associated to the discrete states. There are indeed correction terms that do not scale like the volume (of the cigar, or the cap of the cigar). These terms are found by expanding the partition function in $\tau_{2} / k$ around the origin. The leading correction would seem to be

$$
Z_{1}=\frac{1}{2 \pi} \frac{1}{|\eta|^{4}} \partial_{\tau}\left(\frac{\left|\theta_{3}^{4}-\theta_{4}^{4}-\theta_{2}^{4}\right|^{2}}{|\eta|^{8}}\right) \int d^{2} x \frac{\left(x_{1}+i x_{2}\right)^{2}}{\left|x_{1}+i x_{2}\right|}+\text { c.c. }
$$

Ignoring for a moment the integral over $x_{1}$ and $x_{2}$, this term is exactly the non-modular invariant contribution of the discrete states in the large $k$ limit found in [5] (see equation (5.17) in that paper and the relation between the two in appendix A.2.1). The contribution in equation (5.18), however, vanishes due to the angular integration. It is thereby trivially modular invariant.

We encounter a puzzling situation. The discrete states make a distinct contribution to the elliptic genus, but they appear to be well hidden in the partition function expression. Despite the vanishing of this particular subleading term, there are two ways to see the imprint of the discrete states on the partition function. The first way is to calculate the next term in the expansion of the partition function. The second way is to turn on R-charge chemical potential $\alpha$ and/or angular momentum chemical potential $\beta$. We hope to return to these avenues in the near future.

\section{A UV/IR relation}

From our results in section 5, it is clear that the relationship between the holonomy space $\left(x_{1}, x_{2}\right)$ and the target space is an ultraviolet/infrared map. Below, we discuss this relation and use it in order to locate the discrete states in the target space. This is an alternative to the wave-function analysis of subsection 3.3, to pinpoint where these states live. We show that the UV/IR relation supports our conclusion that these states are located at the string scale tip of the cigar.

The fact that there is a UV/IR relation between the holonomy space and the target space is most easily seen from the divergences we encountered in the partition functions. In the holonomy space, we cut out a small disk of radius $\varepsilon$ around each pole of the integrand, which roughly corresponds in the target space to a cutoff on the semi-infinite cylinder (see the figures). The smaller the cut-off $\varepsilon$ is, the larger the part of the volume of the cylinder that we take into account is. This illustrates the UV/IR nature of the relationship between 
the holonomy space and the target space. Note that the little disk need not be around the origin for this to be true, as is clear from the discussion about the non-zero winding sector (around equation (5.17)). In a sense, this UV/IR relation is like a Fourier transformation.

We wish to take advantage of this UV/IR relation in order to revisit the location of the discrete states in the target space. It is clear they do not live in the semi-infinite cylinder. The question is whether they are located at the tip or the whole cap of the cigar.

With this motivation in mind, let us consider the elliptic genus. It has two pieces. The non-holomorphic term is due to the difference in spectral densities $\Delta \rho$ in the continuum. As emphasized in section 2 , the spectral asymmetry $\Delta \rho$ is fixed by the asymptotic rightmoving supercharge. The asymptotic region is described by supergravity and so the relevant distance from the tip is $\sqrt{k} l_{s}$, which is the only scale available at the supergravity level. In the holonomy space, we saw that the contribution to the non-holomorphic piece comes from a region of size $\sim 1 / \sqrt{k}$. We find, therefore, that in string units

$$
\frac{1}{\sqrt{k}} \text { in holonomy space } \Leftrightarrow \sqrt{k} \text { in target space. }
$$

The holomorphic piece in the elliptic genus and in the partition function originate in a region of size $\sim 1$ in the holonomy space. The UV/IR relation thus implies that they are located, in target space, at a distance scale from the tip which is a scale smaller than $\sqrt{k}$. The only other scale in the problem is the string scale. This suggests that

$$
1 \text { in holonomy space } \Leftrightarrow 1 \text { in target space, }
$$

and that the discrete states live at the tip of the cigar.

Twisted sectors as a ruler. To make our argument sharper still, we consider the elliptic genus of the cigar orbifolded by a $\mathbb{Z}_{M}$ subgroup of the R-symmetry group. We take $k \rightarrow \infty$ while keeping the order $M$ finite. Now, there are also twisted sectors in the theory. These can be used as a ruler that relates distances in the target space to distances in holonomy space. These twisted degrees of freedom live a stringy distance away from the tip. To determine their size in holonomy space, we calculate the elliptic genus in this case. The untwisted and twisted sectors are parameterized by two integers, $p_{1}, p_{2}=0,1,2 \ldots, M-1$, and their contribution to the elliptic genus is

$$
\begin{aligned}
\chi_{\cos }^{M}(\tau, \alpha)= & \frac{k}{M} \sum_{p_{1}, p_{2} \in \mathbb{M}} \int_{0}^{1} d s_{1,2} \sum_{m, w \in \mathbb{Z}} \frac{\theta_{11}\left(s_{1} \tau+s_{2}-\frac{k+1}{k} \alpha, \tau\right)}{\theta_{11}\left(s_{1} \tau+s_{2}-\frac{\alpha}{k}, \tau\right)} \\
& \times e^{2 \pi i \alpha w} e^{-\frac{k \pi}{\tau_{2}}\left|\left(m+\frac{p_{1}}{M}+s_{2}\right)+\left(w+\frac{p_{2}}{M}+s_{1}\right) \tau\right|^{2}} .
\end{aligned}
$$

We can again rewrite this as an integral over the whole complex plane,

$$
\begin{aligned}
& \chi_{\cos }^{M}(\tau, \alpha)= \\
& \frac{1}{M} \sum_{p_{1}, p_{2} \in \mathbb{M}} \int d^{2} x \frac{\theta_{11}\left(\sqrt{\frac{\tau_{2}}{k}}\left(x_{1}+i x_{2}\right)-\frac{k+1}{k} \alpha, \tau\right)}{\theta_{11}\left(\sqrt{\frac{\tau_{2}}{k}}\left(x_{1}+i x_{2}\right)-\frac{\alpha}{k}, \tau\right)} e^{-\pi\left(\left(x_{1}+\frac{p_{1}}{M} \sqrt{\frac{k}{\tau_{2}}}+\frac{p_{2}}{M} \sqrt{\frac{k}{\tau_{2}}} \tau_{1}\right)^{2}+\left(x_{2}+\frac{p_{2}}{M} \sqrt{k \tau_{2}}\right)^{2}\right)},
\end{aligned}
$$


from which we see that the twisted sector contribution is coming from a region of size $\sim 1$, located around $\left(x_{1}, x_{2}\right)=-\left(\frac{p_{1}}{M} \sqrt{\frac{k}{\tau_{2}}}+\frac{p_{2}}{M} \sqrt{\frac{k}{\tau_{2}}} \tau_{1}, \frac{p_{2}}{M} \sqrt{k \tau_{2}}\right)$.

We see that, indeed, regions of size of order 1 in holonomy space correspond to the tip of the cigar. Again, this supports our claim that the discrete states also live at the tip of the cigar.

\section{The trumpet and universality}

In this section, we clarify a few aspects of the universality discussed in the previous sections. Note that the Wick rotation of the region beyond the singularity of the $\operatorname{SL}(2, \mathbb{R})_{k} / \mathrm{U}(1)$ black hole yields the trumpet geometry; it has an exact conformal field theory description in terms of a $\mathbb{Z}_{k}$ orbifold of the cigar (see e.g. [34] for a review). We study its elliptic genus in the large level limit.

The elliptic genus of the trumpet is

$$
\chi_{\mathrm{orb}}=\chi_{\mathrm{orb}}^{\mathrm{hol}}+\chi_{\mathrm{orb}}^{\mathrm{rem}}
$$

with [1]

$$
\begin{aligned}
\chi_{\mathrm{orb}}^{\mathrm{hol}} & =\frac{i \theta_{11}(z, q)}{\eta^{3}} \sum_{m \in \mathbb{Z}} \frac{q^{k m^{2}} z^{2 m}}{1-z^{\frac{1}{k}} q^{m}} \\
\chi_{\mathrm{orb}}^{\mathrm{rem}} & =-\frac{i \theta_{11}(z, q)}{\pi \eta^{3}} \sum_{p, w} \int_{-\infty-i \epsilon}^{+\infty-i \epsilon} \frac{d s}{2 i s-p+k w} q^{\frac{s^{2}}{k}+\frac{(k w+p)^{2}}{4 k}} z^{-\frac{k w+p}{k}} \bar{q}^{\frac{s^{2}}{k}+\frac{(k w-p)^{2}}{4 k}} .
\end{aligned}
$$

Following the logic of section 4, we focus first on the non-holomorphic piece, from which one may determine the holomorphic piece via universality and modularity. In the large $k$ limit, the non-holomorphic piece does not depend on the level $k$; it reads

$$
\chi_{\mathrm{orb}}^{\mathrm{non}-\mathrm{hol}}(k=\infty)=-\frac{i \theta_{11}}{\eta^{3}} \frac{i \alpha}{2 \tau_{2}} .
$$

In particular, it is minus the non-holomorphic piece of the cigar. It is also determined by the asymptotic supersymmetry and cylindrical topology. For the trumpet's throat, the sign of the spectral asymmetry $\Delta \rho$ is opposite to that of the cigar.

In section 4, we used modularity as well as the assumption of a smooth filling in of the geometry to argue that the holomorphic piece is universal as well. Now, the trumpet background is not in the same universality class as the cigar; the topology is different, and the string coupling diverges at the singularity. In its weakly coupled T-dual description, in terms of the $\mathbb{Z}_{k}$ orbifold of the cigar, it has an orbifold fixed point at the tip. Hence, the near tip background behaves like a $\mathbb{Z}_{k}$ orbifold of $\mathbb{R}^{2}$, and so its Witten index is $k$ (which contrasts with the cigar with Witten index 1). The orbifold singularity thus sets a new interior boundary condition for the allowed filling-in. It implies that the elliptic genus scales like the level $k$. Indeed, the holomorphic piece in the large level limit is

$$
-\frac{i \theta_{11}}{\eta^{3}} \frac{k}{2 \pi i \alpha}
$$


This expression is modular covariant by itself for a theory with central charge $c=3$. However, the central charge here is $c=3+6 / k$. The difference, $6 / k$, goes to zero, but since the result (7.4) scales like $k$, this difference has a finite effect on the modular covariance properties of (7.4) (see equation (3.2)), even when $k \rightarrow \infty$. This finite effect is exactly canceled against that of the non-holomorphic piece (7.3). That is,

$$
\chi_{\mathrm{orb}}(k=\infty)=-\frac{i \theta_{11}}{\eta^{3}}\left(\frac{k}{2 \pi i \alpha}+\frac{i \alpha}{2 \tau_{2}}\right)
$$

is modular covariant with $c=3+6 / k$.

A potentially interesting way to think about the diverging order $k$ contribution to the elliptic genus of the trumpet, is to add to it the elliptic genus corresponding to the Wick rotation of the interior of the black hole - the regime between its horizon and singularity - which is equal to [35]

$$
\chi_{\text {interior }}=-\chi_{M M}(-k)
$$

where

$$
\chi_{M M}(k)=\frac{\theta_{11}\left(z^{1-\frac{1}{k}}, q\right)}{\theta_{11}\left(z^{\frac{1}{k}}, q\right)}
$$

is the elliptic genus of the $\mathrm{SU}(2)_{k} / \mathrm{U}(1)$ superconformal field theory - an $N=2$ minimal model at level $k$. The curious sum rule found in [35, 36],

$$
\chi_{\text {orb }}+\chi_{\text {interior }}+\chi_{\text {cigar }}=0,
$$

which is correct for any positive integer level $k$, implies that the interior of the black hole provides an intriguing regularization of the regime beyond the singularity, since the sum of their elliptic genera is indeed (the minus of) the elliptic genus of the cigar. It would be very interesting to see if this holds more generally and, in particular, for Schwarzschild black holes.

\section{Summary and discussion}

In this work, we continued the investigation of perturbative string theory in the background of large Euclidean black holes. The main focus in this paper was to study the elliptic genus in the $\mathrm{SL}(2, \mathbb{R})_{k} / \mathrm{U}(1)$ superconformal field theory in the large level limit and explore its physical meaning. We found that it is finite and contains two pieces. One is holomorphic and is due to the discrete states, the other is non-holomorphic and is due to a mismatch in the densities of worldsheet bosons and fermions in the continuum. This mismatch is a feature that appears already at the gravity level and is fully fixed by the topology of the cigar. In other words, it cannot be avoided in any theory of quantum gravity. Modularity of string theory forces the existence of the holomorphic piece, and hence the existence of the discrete states even for parametrically small curvature.

Although the elliptic genus was analyzed for the exact superconformal background of the two-dimensional black hole, universality and modularity, discussed in this work, indicate 
that the properties above are generic, including for perturbative strings propagating e.g. in the background of a large four-dimensional Schwarzschild black hole.

We addressed the question: "Where in target space do these discrete states live in the large level limit?" in two ways. The answer is that they live at a stringy distance from the tip of the cigar. This, we view, as evidence for the claims made in $[4,5]$ that the tip of the cigar is special in string theory even for parametrically small curvature. There are degrees of freedom in the theory with definite conformal dimension and a wave function that is supported at a stringy distance from the tip. In contrast, in the free scalar conformal field theory associated to $\mathbb{R}^{2}$, states with definite conformal dimension are smeared over the whole plane.

In short, the elliptic genus has enough structure to indicate that perturbative string theory in the background of a large black hole is yet to reveal its full peculiarities. Questions remain, like: "What are the implications, if any, of the degrees of freedom at the tip for an infalling observer?" This, we believe, should be determined by the Wick rotation of the interaction between the tip degrees of freedom and the gravitons, dilaton, et cetera, in the limit where the curvature is small. We hope to make progress on that front as well.

\section{Acknowledgments}

We thank Ofer Aharony, Sujay Ashok, Sameer Murthy and Suresh Nampuri for discussions. J.T. would like to thank the Hebrew University of Jerusalem as well as Tel-Aviv University for their warm hospitality during his visit. The work of A.G. and N.I. is supported in part by the I-CORE Program of the Planning and Budgeting Committee and the Israel Science Foundation (Center No. 1937/12). The work of A.G. is supported in part by the BSF American-Israel Bi-National Science Foundation, and by a center of excellence supported by the Israel Science Foundation (grant number 1665/10).

\section{A Useful formulas}

In this appendix, we collect various formulas that we put to use in the main body of the paper.

\section{A.1 Conventions and properties of theta functions}

Our conventions for theta functions as well as some of the properties of their derivatives, relations to series, et cetera are recalled in the following.

\section{A.1.1 Product and sum formulas}

We use the product and sum formulas for theta functions as well as the eta function:

$$
\begin{aligned}
\eta(q) & =q^{\frac{1}{24}} \prod_{m=1}^{\infty}\left(1-q^{m}\right) \\
\theta_{00}(z, q) & =\prod_{m=1}^{\infty}\left(1-q^{m}\right)\left(1+z q^{m-1 / 2}\right)\left(1+z^{-1} q^{m-1 / 2}\right)=\sum_{n \in \mathbb{Z}} q^{\frac{n^{2}}{2}} z^{n} \\
& =\theta_{3}(z, q)=\theta[0,0](z, q)
\end{aligned}
$$




$$
\begin{aligned}
\theta_{01}(z, q) & =\prod_{m=1}^{\infty}\left(1-q^{m}\right)\left(1-z q^{m-1 / 2}\right)\left(1-z^{-1} q^{m-1 / 2}\right)=\sum_{n \in \mathbb{Z}}(-1)^{n} q^{\frac{n^{2}}{2}} z^{n} \\
& =\theta_{4}(z, q)=\theta[0,1 / 2](z, q) \\
\theta_{10}(z, q) & =\left(z^{\frac{1}{2}}+z^{-\frac{1}{2}}\right) q^{\frac{1}{8}} \prod_{m=1}^{\infty}\left(1-q^{m}\right)\left(1+z q^{m}\right)\left(1+z^{-1} q^{m}\right)=\sum_{n \in \mathbb{Z}} q^{\frac{\left(n-\frac{1}{2}\right)^{2}}{2}} z^{n-\frac{1}{2}} \\
& =\theta_{2}(z, q)=\theta[1 / 2,0](z, q) \\
\theta_{11}(z, q) & =i\left(z^{\frac{1}{2}}-z^{-\frac{1}{2}}\right) q^{\frac{1}{8}} \prod_{m=1}^{\infty}\left(1-q^{m}\right)\left(1-z q^{m}\right)\left(1-z^{-1} q^{m}\right)=-i \sum_{n \in \mathbb{Z}}(-1)^{n} q^{\frac{\left(n-\frac{1}{2}\right)^{2}}{2}} z^{n-\frac{1}{2}} \\
& =-\theta_{1}(z, q)=\theta[1 / 2,1 / 2](z, q),
\end{aligned}
$$

where $q=e^{2 \pi i \tau}$ and $z=e^{2 \pi i \alpha}$.

\section{A.1.2 Properties}

We have the properties:

$$
\begin{aligned}
\theta_{1}(0, \tau) & =0 \\
\partial_{\tau} \theta_{1}(0, \tau) & =0 \\
\partial_{\alpha} \theta_{\sigma \neq 1}(0, \tau) & =0 \\
4 \pi i \partial_{\tau} \theta_{\sigma} & =\partial_{\alpha}^{2} \theta_{\sigma}
\end{aligned}
$$

\section{A.1.3 Shifts}

We can shift arguments of $\theta$ functions using the formula:

$$
\theta[a, b]\left(\alpha+m_{2}+m_{1} \tau, \tau\right)=(-1)^{2 a m_{2}}(-1)^{2 b m_{1}} z^{-m_{1}} q^{-\frac{m_{1}^{2}}{2}} \theta[a, b](\alpha, \tau)
$$

in a notation where $a, b$ are zero or one half.

\section{A.1.4 Derivatives}

We can use the product formulae for the theta functions to obtain their derivatives. Their $\alpha$-derivatives are:

$$
\begin{aligned}
\partial_{\alpha} \theta_{00}(z, q) & =\theta_{00}(z, q) \sum_{m=1}^{\infty} 2 \pi i\left(\frac{z q^{m-1 / 2}}{1+z q^{m-1 / 2}}-\frac{z^{-1} q^{m-1 / 2}}{1+z^{-1} q^{m-1 / 2}}\right) \\
& =\theta_{00}(z, q)\left(z-z^{-1}\right) \sum_{m=1}^{\infty} 2 \pi i \frac{q^{m-1 / 2}}{\left(1+z q^{m-1 / 2}\right)\left(1+z^{-1} q^{m-1 / 2}\right)} \\
\partial_{\alpha} \theta_{01}(z, q) & =\theta_{01}(z, q) \sum_{m=1}^{\infty} 2 \pi i\left(\frac{-z q^{m-1 / 2}}{1-z q^{m-1 / 2}}+\frac{z^{-1} q^{m-1 / 2}}{1-z^{-1} q^{m-1 / 2}}\right) \\
& =\theta_{01}(z, q)\left(z^{-1}-z\right) \sum_{m=1}^{\infty} 2 \pi i \frac{q^{m-1 / 2}}{\left(1-z q^{m-1 / 2}\right)\left(1-z^{-1} q^{m-1 / 2}\right)}
\end{aligned}
$$




$$
\begin{aligned}
\partial_{\alpha} \theta_{10}(z, q) & =\theta_{10}(z, q)\left(\pi i \frac{z^{\frac{1}{2}}-z^{-\frac{1}{2}}}{z^{\frac{1}{2}}+z^{-\frac{1}{2}}}+\sum_{n=1}^{\infty} 2 \pi i\left(\frac{z q^{n}}{1+z q^{n}}-\frac{z^{-1} q^{n}}{1+z^{-1} q^{n}}\right)\right) \\
& =\theta_{10}(z, q) 2 \pi i\left(\frac{1}{2} \frac{1-z}{z+1}+\left(z-z^{-1}\right) \sum_{m=1}^{\infty} \frac{q^{n}}{\left(1+z q^{n}\right)\left(1+z^{-1} q^{n}\right)}\right) \\
\partial_{\alpha} \theta_{11}(z, q) & =\theta_{11}(z, q)\left(\pi i \frac{z^{\frac{1}{2}}+z^{-\frac{1}{2}}}{z^{\frac{1}{2}}-z^{-\frac{1}{2}}}+\sum_{n=1}^{\infty} 2 \pi i\left(-\frac{z q^{n}}{1-z q^{n}}+\frac{z^{-1} q^{n}}{1-z^{-1} q^{n}}\right)\right) \\
& =\theta_{11}(z, q) 2 \pi i\left(\frac{1}{2} \frac{1+z}{z-1}+\left(z^{-1}-z\right) \sum_{m=1}^{\infty} \frac{q^{n}}{\left(1-z q^{n}\right)\left(1-z^{-1} q^{n}\right)}\right)
\end{aligned}
$$

\section{A.2 Further properties applied}

Particular applications of these properties, as well as further formulas of use are the following.

\section{A.2.1 Infinite series, theta functions and a partition sum}

In [5], a contribution to the partition function was identified proportional to various infinite series (related to sums of odd divisors). Those series can be rewritten in terms of derivatives of $\theta$ functions as follows:

$$
\begin{aligned}
\Sigma_{R} & =\sum_{n=0}^{\infty} \frac{n q^{n}}{1+q^{n}}=\frac{1}{48}\left(\theta_{3}(q)^{4}+\theta_{4}(q)^{4}-1\right)=\frac{1}{2} q \partial_{q} \log \frac{q^{-\frac{1}{12}} \theta_{2}(q)}{\eta} \\
\Sigma_{N S} & =\sum_{n=0}^{\infty}(2 n+1) \frac{q^{n+1 / 2}}{1+q^{n+1 / 2}}=\frac{\theta_{2}(q)^{4}-\theta_{4}(q)^{4}+1}{24}=q \partial_{q} \log \frac{q^{\frac{1}{24}} \theta_{3}}{\eta} \\
\Sigma_{\tilde{N} S} & =\sum_{n=0}^{\infty}(2 n+1) \frac{q^{n+1 / 2}}{1-q^{n+1 / 2}}=\frac{\theta_{2}(q)^{4}+\theta_{3}(q)^{4}-1}{24}=-q \partial_{q} \log \frac{q^{\frac{1}{24}} \theta_{4}}{\eta} .
\end{aligned}
$$

These equations are the main ingredients in putting the result of [5] in the form of equation (5.18). We also made use of the relation:

$$
\eta(q)=\left(\frac{\partial_{\alpha} \theta_{11}(0, \tau)}{-2 \pi}\right)^{\frac{1}{3}}
$$

as well as:

$$
-2 \pi i \partial_{\tau} \log \eta=\frac{\pi^{2}}{6} E_{2}
$$

where $E_{2}$ is the second Einsenstein series, whose modular completion is

$$
\hat{E}_{2}=E_{2}-\frac{3}{\pi \tau_{2}}
$$

which imply that:

$$
\partial_{\tau} \log \left(\tau_{2}|\eta|^{4}\right)=\frac{1}{2 i \tau}+\frac{i \pi}{6} E_{2}=\frac{i \pi}{6} \hat{E}_{2}
$$




\section{A.2.2 The expansion of the large level genus near $\alpha=0$}

The link between the second Eisenstein series and the derivative of theta functions (near $\alpha=0$ ) can be found either through the manipulations:

$$
E_{2}(\tau)=1-24 \sum_{n=1}^{\infty} \frac{n q^{n}}{1-q^{n}}=1-24 \sum_{n=1}^{\infty} \sum_{m=0}^{\infty} n q^{n(m+1)}=1-24 \sum_{m=1}^{\infty} \frac{q^{m}}{\left(1-q^{m}\right)^{2}}
$$

and observing that this series appears in equation (A.5), or via expansion near $\alpha=0$, the heat equation (A.3) as well as equation (A.8). Using these formulas, we can for instance describe the second term in the expansion of the large level limit of the cigar elliptic genus in terms of $\alpha$. Near $\alpha=0$, the cigar elliptic genus contains the terms:

$$
\chi(k=\infty) \approx \frac{\theta_{11}(\alpha, \tau)}{\eta^{3}(q)}\left(-\frac{1}{2 \pi \alpha}+\alpha \frac{\pi}{6} \hat{E}_{2}\right) \approx 1-\alpha^{2} \frac{\pi^{2}}{2} E_{2}+\frac{\pi \alpha^{2}}{\tau_{2}} .
$$

It can be shown that this expression has the right modular covariance near $\alpha=0$, using the transformation rule for the elliptic genus as well as the (modularly completed) second Eisenstein series $\hat{E}_{2}$.

Open Access. This article is distributed under the terms of the Creative Commons Attribution License (CC-BY 4.0), which permits any use, distribution and reproduction in any medium, provided the original author(s) and source are credited.

\section{References}

[1] J. Troost, The non-compact elliptic genus: mock or modular, JHEP 06 (2010) 104 [arXiv: 1004.3649] [INSPIRE].

[2] T. Eguchi and Y. Sugawara, Non-holomorphic Modular Forms and $\mathrm{SL}(2, \mathbb{R}) / \mathrm{U}(1)$ Superconformal Field Theory, JHEP 03 (2011) 107 [arXiv:1012.5721] [InSPIRE].

[3] S.K. Ashok and J. Troost, A Twisted Non-compact Elliptic Genus, JHEP 03 (2011) 067 [arXiv: 1101.1059] [INSPIRE].

[4] A. Giveon and N. Itzhaki, String Theory Versus Black Hole Complementarity, JHEP 12 (2012) 094 [arXiv: 1208.3930] [INSPIRE].

[5] A. Giveon and N. Itzhaki, String theory at the tip of the cigar, JHEP 09 (2013) 079 [arXiv: 1305.4799] [INSPIRE].

[6] D. Kutasov, Accelerating branes and the string/black hole transition, hep-th/0509170 [INSPIRE].

[7] T.G. Mertens, H. Verschelde and V.I. Zakharov, Random Walks in Rindler Spacetime and String Theory at the Tip of the Cigar, JHEP 03 (2014) 086 [arXiv:1307.3491] [INSPIRE].

[8] R. Dijkgraaf, H.L. Verlinde and E.P. Verlinde, String propagation in a black hole geometry, Nucl. Phys. B 371 (1992) 269 [inSPIRE].

[9] S.K. Ashok, S. Nampuri and J. Troost, Counting Strings, Wound and Bound, JHEP 04 (2013) 096 [arXiv: 1302.1045] [INSPIRE].

[10] E. Witten, Constraints on Supersymmetry Breaking, Nucl. Phys. B 202 (1982) 253 [INSPIRE]. 
[11] R. Akhoury and A. Comtet, Anomalous Behavior of the Witten Index: Exactly Soluble Models, Nucl. Phys. B 246 (1984) 253 [InSPIRE].

[12] E. Witten, Elliptic Genera and Quantum Field Theory, Commun. Math. Phys. 109 (1987) 525 [INSPIRE].

[13] S.P. Zwegers, Mock Theta Functions, Ph.D. Thesis, Utrecht The Netherlands (2002).

[14] D. Zagier, Ramanujan's mock theta functions and their applications (after Zwegers and Ono-Bringmann), Séminaire Bourbaki. Vol. 2007/2008, Astérisque 326 (2009) 143.

[15] S. Elitzur, A. Forge and E. Rabinovici, Some global aspects of string compactifications, Nucl. Phys. B 359 (1991) 581 [INSPIRE].

[16] G. Mandal, A.M. Sengupta and S.R. Wadia, Classical solutions of two-dimensional string theory, Mod. Phys. Lett. A 6 (1991) 1685 [inSPIRE].

[17] E. Witten, On string theory and black holes, Phys. Rev. D 44 (1991) 314 [InSPIRE].

[18] A. Giveon, A. Konechny, A. Pakman and A. Sever, Type 0 strings in a $2-d$ black hole, JHEP 10 (2003) 025 [hep-th/0309056] [INSPIRE].

[19] A. Giveon and D. Kutasov, Little string theory in a double scaling limit, JHEP 10 (1999) 034 [hep-th/9909110] [inSPIRE].

[20] J.M. Maldacena and H. Ooguri, Strings in $A d S_{3}$ and $\mathrm{SL}(2, \mathbb{R}) W Z W$ model 1.: The Spectrum, J. Math. Phys. 42 (2001) 2929 [hep-th/0001053] [InSPIRE].

[21] V.A. Fateev, A.B. Zamolodchikov and Al.B. Zamolodchikov, unpublished.

[22] J. Teschner, Operator product expansion and factorization in the $H+(3)$ WZNW model, Nucl. Phys. B 571 (2000) 555 [hep-th/9906215] [INSPIRE].

[23] K. Hori and A. Kapustin, Duality of the fermionic $2-D$ black hole and $N=2$ Liouville theory as mirror symmetry, JHEP 08 (2001) 045 [hep-th/0104202] [INSPIRE].

[24] E. Kiritsis, C. Kounnas and D. Lüst, A Large class of new gravitational and axionic backgrounds for four-dimensional superstrings, Int. J. Mod. Phys. A 9 (1994) 1361 [hep-th/9308124] [INSPIRE].

[25] K. Hori and A. Kapustin, World sheet descriptions of wrapped NS five-branes, JHEP 11 (2002) 038 [hep-th/0203147] [INSPIRE].

[26] S.K. Ashok and J. Troost, Elliptic genera and real Jacobi forms, JHEP 01 (2014) 082 [arXiv: 1310.2124] [INSPIRE].

[27] S.K. Ashok, N. Doroud and J. Troost, Localization and real Jacobi forms, arXiv:1311.1110 [INSPIRE].

[28] S. Murthy, A holomorphic anomaly in the elliptic genus, arXiv:1311.0918 [INSPIRE].

[29] O. Aharony, A. Giveon and D. Kutasov, LSZ in LST, Nucl. Phys. B 691 (2004) 3 [hep-th/0404016] [INSPIRE].

[30] J. Scherk and J.H. Schwarz, Spontaneous Breaking of Supersymmetry Through Dimensional Reduction, Phys. Lett. B 82 (1979) 60 [INSPIRE].

[31] Y. Sugawara, Thermodynamics of Superstring on Near-extremal NS5 and Effective Hagedorn Behavior, JHEP 10 (2012) 159 [arXiv:1208.3534] [INSPIRE]. 
[32] A. Hanany, N. Prezas and J. Troost, The Partition function of the two-dimensional black hole conformal field theory, JHEP 04 (2002) 014 [hep-th/0202129] [INSPIRE].

[33] D. Israel, C. Kounnas, A. Pakman and J. Troost, The Partition function of the supersymmetric two-dimensional black hole and little string theory, JHEP 06 (2004) 033 [hep-th/0403237] [INSPIRE].

[34] A. Giveon, M. Porrati and E. Rabinovici, Target space duality in string theory, Phys. Rept. 244 (1994) 77 [hep-th/9401139] [INSPIRE].

[35] A. Giveon, N. Itzhaki and J. Troost, The Black Hole Interior and a Curious Sum Rule, JHEP 03 (2014) 063 [arXiv: 1311.5189] [INSPIRE].

[36] Y. Sugawara, "Analytic continuation" of $\mathcal{N}=2$ minimal model, PTEP 2014 (2013) 043B02 [arXiv: 1311.4708] [INSPIRE]. 